\title{
A Big Data Approach to Myocyte Membrane Analysis: Using Populations of Models to Understand the Cellular Causes of Heart Failure
}

\author{
Carlos A. Ledezma ${ }^{1}$, Benjamin Kappler ${ }^{2}$, Veronique Meijborg ${ }^{3}$, Bas Boukens ${ }^{3}$, Marco Stijnen ${ }^{2}$, \\ PJ Tan ${ }^{1}$, Vanessa Díaz-Zuccarini ${ }^{1}$ \\ ${ }^{1}$ Department of Mechanical Engineering, University College London, London, United Kingdom \\ ${ }^{2}$ LifeTec Group, Eindhoven, Netherlands \\ ${ }^{3}$ Academic Medical Center, Amsterdam, Netherlands
}

\begin{abstract}
Experimentally-calibrated populations of models (ePoMs) allow to elucidate the trends hidden behind large amounts of data, but they have never been used as a means to monitor the temporal evolution of a heart's electrical activity. This work aimed at using ePoMs to understand the cell membrane anomalies that lead to heart failure. A population of the Tusscher-Noble-Noble-Panfilov model was calibrated to the activation-recovery intervals measured from epicardial electrograms acquired during a Physioheart experiment. A Mann-Whitney-Wilcoxon U-test was performed on the statistical distributions of the calibrated parameters, at different time points during the experiment, to elucidate the physiology changes that would have led to the resulting ePoMs. The methodology developed in this paper could detect the specific pathological ion dynamics responsible for the abnormal electrical behavior observed during the experiment. Furthermore, the analysis of the electrical activities was capable of detection of pathologies at an earlier stage when compared to the analysis of the cardiac output alone. The use of big data analytics proved to be more effective than the traditional signal analysis approach in predicting heart failure; additionally, this approach accounts for variabilities in both the healthy and the pathological conditions.
\end{abstract}

\section{Introduction}

Cardiovascular diseases remain, to date, the leading cause of death worldwide. It is a challenge for the scientific community to develop methods for their early detection. Since the early 1950s various mathematical models, that describe the ion dynamics of cardiac cells, have been used to study the behavior of the heart. Notwithstanding, there remain significant challenges: on how to accurately represent pathological structures and functions, on a general lack of validation against reliable experimen- tal data [1] and on how to account for inter-subject variability [2]. "Experimentally-calibrated population of models" (ePoM) is a methodology capable of capturing intersubject variability and relies on experimental measurements to characterize pathology [2]. Studies have shown that the ePoM can be employed to elucidate which cellular level processes influence atrial and ventricular pathological behavior. A key limitation of existing ePoM studies is that they had to rely on human datasets, acquired over a short time duration, during coronary bypass or valve replacement surgery; consequently, the sets of parameters that characterize diseased individuals, obtained through the ePoM process, had to be compared to the 'averaged' parameters used in the mathematical models, assumed those for healthy individuals; also, it was impossible to identify the long-term electrophysiological changes accompanying heart failure, or to account for inter-subject variability in the healthy condition.

The Physioheart platform [3], developed by LifeTec Group, is an experimental development where a porcine heart is monitored continuously, over several hours, under an acute setting of functional and hemodynamical conditions that match closely those of an in-vivo exploration. Simultaneously, various data, under different operating conditions, can also be acquired throughout. The countless relationships possible between the several acquisitions available from a Physioheart experiment make it impossible for the naked eye to monitor such a platform, whereas a big data approach - such as the ePoM - may be more suited. A methodology that uses the aforesaid dataset to identify malfunctions in the cell membrane was developed and is presented here. This study is part an on-going research in applying mathematical models and machine learning techniques to elucidate the different cellular level mechanisms that result in organ level pathological behaviors. 


\section{Materials and Methods}

The experimental data reported here was acquired during a Physioheart experiment following the inclusion criteria and ethical approval described by previous authors [3]. During this experiment the porcine heart produced a maximal cardiac output of $4 \mathrm{~L} / \mathrm{min}$, during which the platform was said to be in a normal working mode; when the cardiac output of the heart dropped below $3 \mathrm{~L} / \mathrm{min}$, the 'end-point' of the experiment was said to be reached. The heart was paced using a right ventricular outer tract stimulus with $600 \mathrm{~ms}$ cycle length. During the working mode, surface epicardial electrograms (EGM) were acquired alongside blood samples and measurements allowing the calculation of cardiac output. Measurements began 90 minutes into the start of the working mode to allow for the coordination of data acquisitions, systems setup and stabilization of the heart. The electrical activity was measured by means of 121 electrodes, ordered in a $11 \times 11$ equally-spaced square grid, stitched over the left ventricular wall. The data was recorded by means of a BioSemi ActiveTwo acquisition and pre-processing system, a summary of the recordings can be observed in Table 1.

Table 1. Data collected at each 'time point'. In parentheses the minutes after the beginning of the working mode.

\begin{tabular}{lcr}
\hline \hline Time point & Record duration & Cardiac Output \\
\hline $1(90 \mathrm{~min})$ & $92 \mathrm{~s}$ & $3.8 \mathrm{~L} / \mathrm{min}$ \\
$2(120 \mathrm{~min})$ & $77 \mathrm{~s}$ & $3.7 \mathrm{~L} / \mathrm{min}$ \\
$3(150 \mathrm{~min})$ & $44 \mathrm{~s}$ & $2.7 \mathrm{~L} / \mathrm{min}$ \\
\hline
\end{tabular}

A dataset similar to the one acquired in the current Physioheart experiment has never previously been applied to any ePoM study. The design of the electrode grid, stitched into place, made it possible to record the electrical activity on the epicardial surface, at the same sites, for over an hour in the working mode. This allowed spatial differences in electrical characteristics to be distinguished as the condition of the heart became pathological with time. The activation-recovery interval (ARI), a surrogate measure for action potential duration at $90 \%$ repolarization (APD90) [4], was adopted here as a measure to characterize the electrical activity. The acquired signals were filtered with a band-pass filter, with $f_{1}=0.5 \mathrm{~Hz}$ and $f_{2}=40 \mathrm{~Hz}$, to keep only the interesting frequency components of the EGM. On the filtered signal, the activation time was identified as the point where the derivative of the EGM was at a minimum during the QRS complex and the recovery time was measured from where the signal derivative reached its maximum during the T-wave [4], this is illustrated in Fig. 1. Each signal was characterized by the mean $\left(\mu_{A R I}\right)$ and standard deviation $\left(\sigma_{A R I}\right)$ of its ARIs.

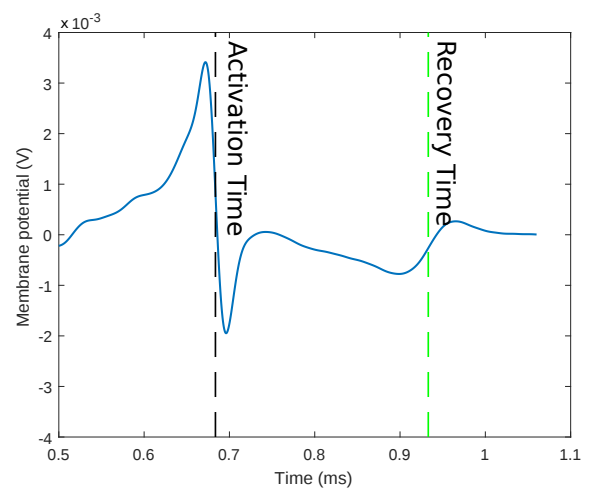

Figure 1. A typical beat's activation and recovery time.

The Tusscher-Noble-Noble-Panfilov (TNNP) model for human ventricular myocytes [5] was adopted to simulate the electrical behavior of the cardiac cell membrane. The TNNP model was chosen because it provides an accurate representation of all the major ionic currents associated with the cellular membrane, it is amenable to efficient implementation compared to others, has been thoroughly validated and is still widely used for a variety of novel applications. All the main peak conductances and currents, normally parameters of the model, were multiplied by $N$ linearly spaced steps between 0 and 2 , where $N$ was the number of models in the population, to generate a space of possible parameters; the use of these multiplying factors ensures both an overexpression and the reduction of the parameters [2]. A population of models (PoM), with $N=15000$ models, was generated by selecting parameter combinations, from the parameter space, using Latin Hypercube Sampling (LHS), which has been proven to capture the full variability of a dataset [6]. Each model was stimulated at $600 \mathrm{~ms}$ cycle length until a steady state response was reached, at which point the APD90 was measured and saved for calibration, to be explained later. Not all models were used in the study, because some parameter combinations produced un-physiological action potentials a model was excluded from the study if the APD90 was longer than $400 \mathrm{~ms}$, if the APD90 was shorter than $100 \mathrm{~ms}$, if the action potential maximum value was less than $0 \mathrm{~V}$, if its resting potential was greater than $-64 \mathrm{mV}$, if its resting potential was less than $-100 \mathrm{mV}$ or if the upstroke time exceeded $10 \mathrm{~ms}$. The PoM after applying the aforesaid criteria was called the corrected PoM.

The corrected PoM was next compared to the experimental dataset, only those models that exhibited an APD90 in accordance with the measured ARIs were retained, this new population set is referred to as an ePoM. Since LHS guarantees that the parameter space is fully represented, the models in an ePoM are all the possible physiological conditions that could have led to the observed ARIs. Since cardiac damage and ion unbalances may occur in isolation 
Table 2. Automatic ARI measurement results, all in ms.

\begin{tabular}{ccccc}
\hline \hline Time-point & $\mu$ & $\sigma$ & $\max$ & $\min$ \\
\hline 1 & $258.5 \mathrm{~ms}$ & $6.6 \mathrm{~ms}$ & $276.4 \mathrm{~ms}$ & $219.2 \mathrm{~ms}$ \\
2 & $252.8 \mathrm{~ms}$ & $12.5 \mathrm{~ms}$ & $295.9 \mathrm{~ms}$ & $206.1 \mathrm{~ms}$ \\
3 & $235.1 \mathrm{~ms}$ & $38.4 \mathrm{~ms}$ & $285.6 \mathrm{~ms}$ & $100.1 \mathrm{~ms}$ \\
\hline \hline
\end{tabular}

on the epicardium, the local behavior of the epicardial wall was characterized by calibrating the corrected PoM individually to each channel. For a given channel at any given 'time-point', an ePoM was generated by selecting only the models, from the corrected PoM, that exhibit an APD90 in the range $\mu_{A R I} \pm \sigma_{A R I}$ corresponding to that channel at the specific 'time-point'. This produced an ePoM for each channel, at each 'time-point', each ePoM contained around 20 models, each model contained 12 parameters; a big data approach was needed to analyze this amount of data.

A statistical analysis was performed to elucidate the changes in physiology that would have led to the resulting ePoMs. Since the ePoMs are characterized by the distributions of their parameters, a Mann-Whitney-Wilcoxon U-test was employed to monitor the median value of their distributions, on each channel, as it varied with time; any differences were considered statistically significant if $\rho \leq 0.001$. This test was performed on every parameter, analyzing its median value at all 'time-points', to determine which ones exhibited a statistically significant variation over time. In this study, it could reasonably be assumed that the datasets corresponding to the beginning of the working mode were at a healthy state and a parameter was considered pathological, on a given channel, if it was statistically different to its corresponding baseline counterpart. Identifying the parameters that exhibited a pathological value over several channels allowed to hypothesize which ion channels were most likely responsible for a reduction in cardiac output.

\section{Results}

Table 2 shows the results for the automatic measurement of the ARIs within their respective 'time-points'. The ARI distribution for 'time-point' 1 exhibited a similar electrical behavior over all the electrodes on the patch; this was exemplified by the fact that most of the ARIs were clustered within a $[250,265] \mathrm{ms}$ time interval. As the experiment progressed towards its 'end-point', the spread of the ARI distribution increased dramatically; this is a direct consequence of the degradation of the cardiac electrical system. The distributions for 'time-points' 2 and 3 showed a clear shortening in their respective ARIs, reaching values as low as $100 \mathrm{~ms}$, although none of them surpassed $300 \mathrm{~ms}$.

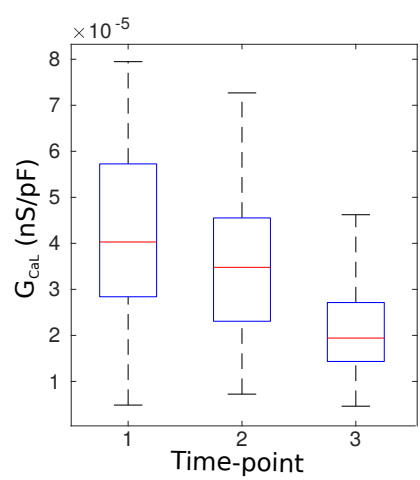

Figure 2. Distribution of $G_{C a L}$ at different time-points for channel 6.

Our results appear to show that an impaired L-type calcium conductance $\left(G_{C a L}\right)$ was mainly responsible for the ARI shortening, it being statistically lower in 61 channels, when comparing time-point 1 and time-point 2 , and in 85 channels, when comparing time-point 1 and time-point 3; all the other parameters showed statistically significant differences in less than 15 channels. Figure 2 shows the main highlight of the ePoM methodology results, it presents the results for only one representative channel, but they were largely similar in the remaining electrodes; it shows a clear steady reduction in the median value of the L-type calcium conductance $\left(G_{C a L}\right)$ with time, this was increasingly evident in the increasing number of channels over time.

The electrolyte concentrations in the blood samples, taken during the Physioheart experiment, revealed a steady increase in calcium and sodium concentration, whereas the concentration of potassium remained largely constant through the working mode. It is worth emphasizing that the ion concentrations already exhibited pathological values from the start of the experiment; this is a limitation of the Physioheart platform. Notwithstanding, the cardiac output $(\geq 3 \mathrm{~L} / \mathrm{min})$ and the ARI values $(\simeq 250 \mathrm{~ms})$ throughout the experiment allowed to assume that the platform was able to model an acute setting of the heart function.

\section{Discussion}

The pathological evolution of the porcine heart was clearly evident from the data summarized in Table 2. Under physiological conditions, and given that the activation is fast and homogeneous, the ARI distribution should be largely similar to that obtained for 'time-point' 1 . This is because all sites on the left ventricle should repolarize in more or less the same time. Together with a stable cardiac output, this supports the choice of using 'time-point' 1 as the healthy benchmark. Over time, the electrical behavior on the epicardial wall became heterogeneous, it was evi- 
dent that the activation-recovery intervals were shortening on the epicardium.

The dataset at 'time point' 2 showed statistically significant differences to 'time-point' 1 . This lead to conclude that the cellular membrane in 'time-point' 2 was not behaving in a physiologically normal manner. This conclusion was not yet reflected in the measured mean cardiac output, which suggests that pathological behavior must first mainfest itself at the cellular scale rather than at an organ scale. A direct consequence of this finding is that this methodology could detect pathologies at an earlier stage, as opposed to a system based on monitoring the cardiac output, used at the present moment. As reflected in Fig. 2, half of the channels showed a significant variation in Ltype calcium conductance after 30 minutes. With time, the reduction in the ARIs was accentuated as more channels showed a pathological $G_{C a L}$. The increase in number of sites exhibiting a pathological behavior was accompanied by an evident reduction in cardiac output. Furthermore, the above suggests that a drop in cardiac output started becoming evident only after a significant number of sites became compromised. Results shown in Fig. 2 suggest that there was a pathological reduction in $G_{C a L}$, this implies that the cell was not absorbing enough calcium as it required to function normally. It is through the L-type calcium channels that the cell receives the calcium needed to activate sarcomere shortening, an augmented resistance to calcium uptake could be a reason why cells lost contractility with time, and cardiac output dropped. The elevated calcium unbalance, since calcium release currents remained unaffected, predicted by the current methodology could also explain the elevated calcium concentration in blood, as this could have been a result of the cell membrane becoming increasingly resistant to calcium as calcium release into the bloodstream remained at the same rate.

A key difference between this and previous studies is that the healthy baseline case was characterized using an ePoM as opposed to using fixed parameters. Also, each electrode was individually monitored, providing insight into the local behavior of the cellular membrane as opposed to the global behavior of the organ. The results shown here suggest that it is possible to identify the specific problems associated with ion uptake or release, that are contributing to heart failure, whilst taking variability into account from the start of the experiment, this is the main contribution of this work.

\section{Conclusions}

An ePoM methodology was developed to monitor the temporal evolution of the electrical behavior of the heart; this was used to identify the specific pathological ion dynamics that contributed to cardiac failure, whilst accounting for variability in both the healthy and the pathologi- cal states. This computational approach successfully identified the generalized impairment in L-type calcium current uptake, which was the most likely cause for reduced contractility in the myocytes. Furthermore, this method was able to detect pathological behaviors at a much earlier stage than currently used methods. These findings were confirmed by the elevated ionized calcium concentration measured in blood samples, a reduction of the myocyte contractility, analysis of the cardiac output and eventual failure of the heart during a Physioheart experiment.

\section{Acknowledgements}

This project has received funding from the European Union's Horizon 2020 research and innovation programme under the Marie Sklodowska-Curie grant agreement No 642612, VPH-CaSE (www.vph-case.eu)

\section{References}

[1] Clayton R, Bernus O, Cherry E, Dierckx H, Fenton F, Mirabella L, Panfilov A, Sachse FB, Seemann G, Zhang H. Models of cardiac tissue electrophysiology: progress, challenges and open questions. Progress in biophysics and molecular biology 2011;104(1):22-48.

[2] Zhou X, Bueno-Orovio A, Orini M, Hanson B, Hayward M, Taggart P, Lambiase PD, Burrage K, Rodriguez B. In vivo and in silico investigation into mechanisms of frequency dependence of repolarization alternans in human ventricular cardiomyocytes. Circulation research 2016;118(2):266-278.

[3] de Hart J, de Weger A, van Tuijl S, Stijnen J, van den Broek $\mathrm{CN}$, Rutten M, de Mol BA. An ex vivo platform to simulate cardiac physiology: a new dimension for therapy development and assessment. The International journal of artificial organs 2011;34(6):495-505.

[4] Potse M, Vinet A, Opthof T, Coronel R. Validation of a simple model for the morphology of the $\mathrm{T}$ wave in unipolar electrograms. American Journal of Physiology Heart and Circulatory Physiology 2009;297(2):H792-H801.

[5] ten Tusscher KH, Panfilov AV. Alternans and spiral breakup in a human ventricular tissue model. American Journal of Physiology Heart and Circulatory Physiology 2006; 291(3):H1088-H1100.

[6] McKay MD, Beckman RJ, Conover WJ. A comparison of three methods for selecting values of input variables in the analysis of output from a computer code. Technometrics 2000;42(1):55-61.

Address for correspondence:

Name: Vanessa Díaz-Zuccarini

Address: Department of Mechanical Engineering, UCL, London, WC1E 7JE

E-mail address: v.diaz@ucl.ac.uk 